\title{
Constructing Strings at the Nano Scale via Staged Self-assembly
}

\author{
Erik D. Demaine \\ Computer Science and Artificial Intelligence Laboratory \\ Massachusetts Institute of Technology \\ 32 Vassar St., Cambridge, MA 02139 \\ edemaine@mit.edu
}

\begin{abstract}
Tile self-assembly is an intriguing approach to manufacturing desired shapes with nano-scale feature size. A recent direction in this theory allows the use of multiple stages - operations performed by the experimenter, such as mixing two self-assembling systems together. This flexibility transforms the experimenter from a passive entity into a parallel algorithm, and vastly reduces the number of distinct parts required to construct a desired shape, possibly making the systems practical to build.

We start with the relatively simple goal of constructing $1 \mathrm{D}$ strings of tiles with distinctive markers, while minimizing the number of mixing steps (work) performed by the staged assembly. In the practical situation of few different "glues", this problem turns out to be closely related to compressing a string into a context-free grammar with the fewest nonterminals. In general, however, the problems turn out to be quite different, as the implicit parallelism of a mixing operation can be exploited to reduce the number of steps.

The staged-assembly perspective also enables the possibility of additional operations, such as adding an enzyme that destroys all tiles with a special label. By enabling destruction in addition to the usual construction, we can perform tasks impossible in a traditional self-assembly system. For example, we can build a Replicator, which transforms a given object of unknown shape or size into many copies of that shape; and we find a vastly more efficient way to construct a nano computer through self-assembly.
\end{abstract}

